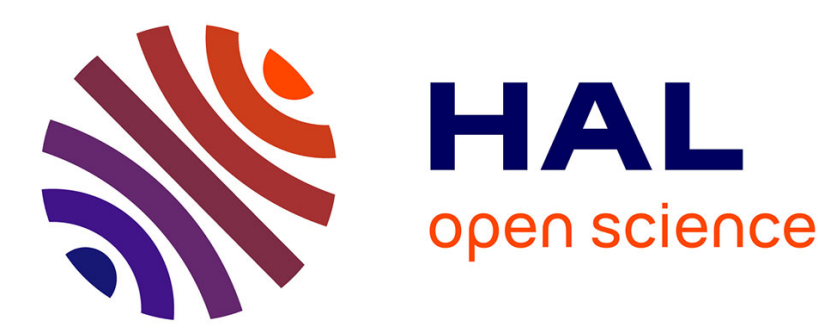

\title{
The role of trip lengths calibration in model-based perimeter control strategies
}

Sergio Batista, Deepak Ingole, Ludovic Leclercq, Monica Menendez

\section{To cite this version:}

Sergio Batista, Deepak Ingole, Ludovic Leclercq, Monica Menendez. The role of trip lengths calibration in model-based perimeter control strategies. IEEE Transactions on Intelligent Transportation Systems, 2021, 11p. 10.1109/TITS.2021.3049679 . hal-03148734

\section{HAL Id: hal-03148734 \\ https://hal.science/hal-03148734}

Submitted on 22 Feb 2021

HAL is a multi-disciplinary open access archive for the deposit and dissemination of scientific research documents, whether they are published or not. The documents may come from teaching and research institutions in France or abroad, or from public or private research centers.
L'archive ouverte pluridisciplinaire HAL, est destinée au dépôt et à la diffusion de documents scientifiques de niveau recherche, publiés ou non, émanant des établissements d'enseignement et de recherche français ou étrangers, des laboratoires publics ou privés. 


\title{
The role of trip lengths calibration in model-based perimeter control strategies
}

\author{
Sérgio Batista, Deepak Ingole, Ludovic Leclercq, and Mónica Menéndez
}

\begin{abstract}
Since the introduction of the Macroscopic Fundamental Diagram (MFD), many traffic control strategies and algorithms have been developed to implement MFD-based perimeter control over a specific urban region. A model-based controller consists of two components: a plant model that represents reality; and a prediction model used to determine optimal control actions. In most studies, the authors assume a constant average trip length for all drivers traveling within the same region, for the prediction model. In these studies about perimeter control and MFD traffic models, the controllers show a good performance because accumulations, i.e. traffic states, from the plant are used to reflect the initial state of the prediction model with a high frequency (about a few seconds). However, this average trip length changes over time as it depends on the OriginDestination flow decomposition, playing an important role in real applications. The main contributions of this paper are twofold. First, we show that the assumption about constant trip lengths used in the prediction model deteriorates the controller's performance for low frequency updates of the optimal control actions. Second, we propose a methodological framework based on the Unscented Kalman Filter (UKF) for dynamically adjusting the average trip lengths and accumulations. Our test results on a real city network show that applying this methodological framework significantly improves the controller's performance.
\end{abstract}

Index Terms-Dynamic trip lengths, Macroscopic Fundamental Diagram traffic models, Nonlinear Model Predictive Control, Unsented Kalman Filter, Multi-regional networks.

\section{INTRODUCTION}

A GGREGATED traffic models attracted more interest from the scientific community after the works of [1] and [2]. These models require the partition of the city network into regions (see e.g., [3]-[7]), where the traffic conditions are considered homogeneous and characterized by a well defined relationship between the mean flow $q_{r}$ and accumulation $n_{r}$ of circulating vehicles, called Macroscopic Fundamental Diagram (MFD). Figure 1 (a) depicts an example of a city network partitioning. The partition defines the borders between two adjacent regions. These borders are located on nodes of the city network, which we refer to as border nodes. Figure 1 (b) shows the regional network gathered from the partitioning, where the vertices of the graph represent the regions. The possible travel directions between adjacent regions are represented by the gray arrows and depend on the city network topology, i.e. on the available travel directions of the incoming and outgoing

Sérgio Batista and M. Menéndez are with the Division of Engineering, New York University Abu Dhabi, United Arab Emirates. e-mail: sergio.batista@nyu.edu; monica.menendez@nyu.edu.

Deepak Ingole and Ludovic Leclercq are with the Univ. Gustave Eiffel, ENTPE, LICIT, Lyon, France. e-mail: deepak.ingole@univ-eiffel.fr; ludovic.leclercq@univ-eiffel.fr links of the border nodes in the city network. Let $X$ be the set of regions that define the regional network.

One of the main challenges for the application of the aggregated MFD traffic models lies in the scale-up of trips in the city network to paths in the regional network. Figure 1 (c) shows one green and two red trips in the city network. One can observe that they cross a different sequence of regions, according to the definition of the city network partitioning. They are then linked to different regional paths. We define as a regional path, the ordered sequence of crossed regions by drivers from the Origin $(\mathrm{O})$ to the Destination $(\mathrm{D})$ regions. The regional paths linked to these trips are depicted in Figure 1 (d). The two red trips cross the same sequence of regions, being then linked to the same red regional path. The green trip crosses a different sequence of regions, and is then linked to the green regional path. (a)

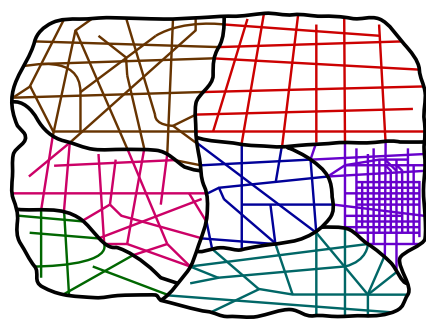

(c)

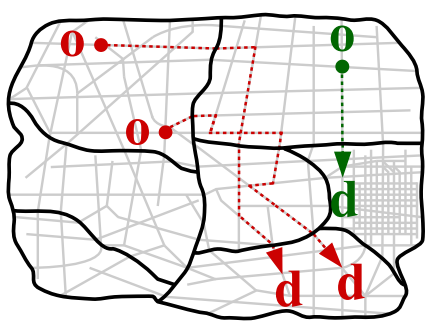

(b)

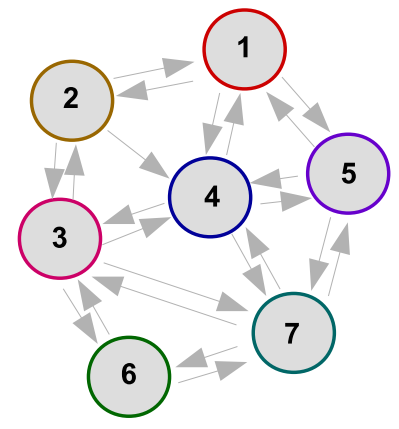

(d)

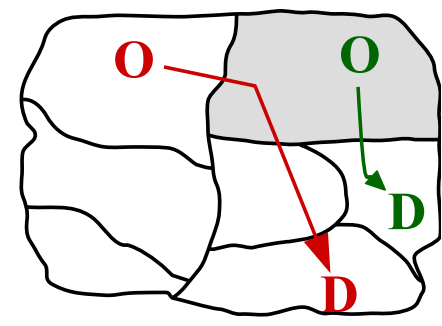

Fig. 1: (a) Example of a partitioned city network. (b) Regional network associated to the partitioning defined in (a). (c) Example of two red and one green trip in the city network. (d) Regional paths associated to the green and red trips.

In reality, as one would expect, different trips associated to the same regional path might have different travel distances in each region. While a trip in the city network is defined by a sequence of links with a fixed physical length, a regional 
path is characterized by a distribution of trip lengths for each region. [8] showed that the calibration of the trip lengths, i.e. the distance that drivers have to travel in each region, clearly influences the traffic dynamics. The authors proposed a methodological framework to calculate these trip length distributions. The framework is based on: (i) the sampling of a large set of virtual trips in the city network corresponding to randomly sampled origin-destination pairs; and (ii) different levels of aggregation following the sequence of regions crossed by these virtual trips. The set of trips can also be obtained from observations of city trip patterns. However, these data are hardly accessible, and a virtual set provides a good proxy. The regional paths are determined by scaling-up these virtual trips according to the sequence of regions they cross. For each regional Origin-Destination (OD) pair, they are ranked from the most to the least significant one. The level of significance is given by the number of virtual trips associated to each regional path. The next step consists in determining the trip length distributions associated to these regional paths. Let $\left\{L_{r p}\right\}$ be the trip length distribution of a generic regional path $p$ in a generic region $r$. These distributions are explicitly calculated according to different levels of information from the city network partitioning. The latter ranges from no prior information about the previous and next regions to be traveled, to a very specific regional path. The first level assigns the same trip length distribution for all regional paths that cross the same region. We refer to this level as the standard or $M_{s}$ for short. The most detailed level of information assigns different trip length distributions to the different regional paths crossing the same region. We refer to this level as the reference or $M_{r}$ for short.

Many of the MFD-based applications have been designed for testing different control algorithms or setting perimeter control strategies (see e.g. [9]-[15] for a few examples). [1] was the first to utilize an optimal control policy and an MFDbased traffic model on a one-region network. This work was later extended by [16] to a 2-regions network. In both of these studies, the authors set a control strategy that maximizes the trip completion rate on the network. [17] was the first to pursue a nonlinear model-predictive control (MPC) approach on a 2-regions network. Nonlinear MPC represents a family of well-known perimeter control models, that are efficient to design control strategies for nonlinear multivariate systems with operating constraints on both input and output variables. They are of particular interest for perimeter control applications, since traffic systems are highly non-linear. Several MPC-based models have been considered in the literature for solving perimeter control problems, such as linear MPC [18], non-linear MPC [14], [17], [19], hybrid MPC [11], [20], hierarchical MPC [9], [21], economic MPC [11], or stochastic MPC [12]. The readers can find comprehensive reviews describing the different MPC formulations in [22][25]. The design of centralized controllers for large-scale urban networks have to account for the (i) non-linear dynamics of the traffic system; (ii) the linear and non-linear constraints arising from traffic inflows and outflows at the perimeter and accumulation of vehicles inside the perimeter; (iii) the possibility to predict future behavior of the system to make better decisions beforehand; and (iv) the optimal use of the network infrastructure to operate at its capacity. These reasons show the suitability of non-linear MPC for perimeter control of traffic systems, and justifies our choice of this kind of controller for this work.

The efficiency of the strategies on optimal control, such as MPC, deteriorate with the increase of noise in the observations, uncertainty on the input parameters (e.g. on the demand or on the MFD itself), or errors associated with model or predictions. Some authors ( [9], [17], [26], [27]) have designed robust control frameworks that are able to handle uncertainty on the demand and MFDs, or stochastic frameworks [12] to deal with such uncertainty in a more flexible manner. On the other hand, most of the existing perimeter control strategies assume perfect knowledge about the accumulation states and inflow demands, requiring information about the Origin and Destinations of drivers. This is unfeasible from a practical perspective. [28] propose a framework that incorporates a joint moving estimation horizon with model predictive perimeter control scheme, to handle noisy observations and the impossibility of measuring state accumulations and inflows per path. To the best of our knowledge, none of the existent studies in the literature has questioned the influence of the trip lengths calibration on the real-time implementation of MPC-oriented perimeter controllers.

A model-based perimeter controller is made of two layers. The plant model consists of an MFD traffic model representing the real traffic states in the network, where detailed trip lengths and path accumulations are used. The optimal approach (i.e. the optimal information level) for calibrating the trip lengths for the plant model, would consider the individual trip length for each driver in the network. However, this is not only unfeasible from a practical perspective, but it would also add a layer of complexity into the modeling framework that is out of the scope of this study. Instead, the reference level assigns different trip lengths for all drivers traveling in the same region, but on different paths. This level is the closest to the individual trip lengths of drivers in the city network [8]. For the purposes of this study, it is the right and sufficient candidate for the plant model. Let $\bar{L}_{r p}$ be the average trip length of regional path $p$ in region $r$, used for the plant model. Following the discussion in [8], we note that the reference level does not change with the dynamics of the traffic states in the network.

The prediction model consists of control decisions that are made based on an MFD traffic model, that accounts for a more aggregated level of information in terms of trip lengths and accumulations per region. In the ideal scenario, the MFD traffic model used for the prediction model would also account for trip lengths and accumulations at the regional path level in each region. However, the reference level is difficult to calibrate for real applications. The main reason is the difficulty in obtaining the full information of drivers' trips, to determine the travel distances according to the reference level, i.e. the calculation of trip lengths by regional path. It is almost impossible to determine in real-time how many vehicles are traveling on each regional path and each region. Only the regions' accumulation can be estimated in practice. [9] 
considered time-dependent trip lengths that are dynamically calculated only based on exchange flows between the regions. This approach neglects important features of the city network topology that are captured by the virtual set of trips [8]. It is therefore unfeasible for control applications in real city networks. As an alternative, most of the studies in the literature (e.g. [11], [12], [17], [18], [26], [27], [29]-[32]) consider a constant average trip length for all drivers in each region $r$, i.e. the standard level, which is easier to calibrate than the reference level. Let $\bar{L}_{r}$ be the average distance to be traveled by all drivers in region $r$, independent of their path $p$. In fact, $\bar{L}_{r}$ changes over time because the number of vehicles circulating on a regional path $p$ and region $r$, i.e. $n_{r p}(t)$, also does [10]. One can estimate $\bar{L}_{r}$ as follows:

$$
\bar{L}_{r}(t)=\frac{\sum_{p \in \Gamma} n_{r p}(t) \bar{L}_{r p} \delta_{r p}}{\sum_{p \in \Gamma} n_{r p}(t) \delta_{r p}}, \forall r \in X
$$

where $\Gamma$ is the set of all regional paths; and $\delta_{r p}$ is a binary variable that equals 1 if regional path $p$ travels on region $r$, or 0 otherwise.

There are two challenges for estimating $\bar{L}_{r}$ using (1). First, it requires the calculation of an average trip length for each path $p$ in region $r\left(\bar{L}_{r p}\right)$. Second, we can only estimate the regions' accumulation $n_{r}$ in practise, as previously mentioned.

The time-independence assumption of $\bar{L}_{r}$ of many studies in the literature has not degraded the controllers' performance because of high frequency updates of the optimal control actions. This means that the accumulations in the prediction model are replaced with direct observations. The main contributions of this paper are twofold. First, we first show that under this assumption of time-independent average trip lengths $\bar{L}_{r}$, the performance of the controller decreases for lower, but more realistic, update frequencies of the control actions. Second, we propose a methodological framework based on the application of an Unscented Kalman Filter (UKF) for dynamically adjusting $\bar{L}_{r}$, and improving the controllers' performance, i.e. maximizing the throughput of the controlled region(s).

The remainder of this paper is organized as follows. In the next section, we discuss the implementation of the NMPC. We then describe the methodological framework for adjusting the average trip lengths dynamically for the prediction model. Afterwards, we test and discuss the results of the application of the proposed methodological framework on a simulation of a real city network. We finalize this paper by outlining the main conclusions.

\section{Methodological FRAMEWork}

\section{A. MFD traffic model for the plant level}

The MFD traffic model used in the plant level represents the ground-truth and keeps track of the vehicles' accumulation on each regional path $p$ traveling on region $r$. The vehicles' accumulation, $n_{r p}$, depends on the balance between the inflow $q_{i n, r p}(t)$ and outflow $q_{o u t, r p}(t)$ :

$$
\frac{d n_{r p}(t)}{d t}=q_{\text {in }, r p}(t)-q_{\text {out }, r p}(t), \forall r \in X \wedge t>0 .
$$

In the literature, one can distinguish between two kinds of MFD traffic models: the accumulation-based [1], [2], [33]; and the trip-based models [33]-[38]. In this paper, we consider the accumulation-based formulation as described in [33].

The effective outflow $q_{o u t, r p}(t)$ of regional path $p$ is determined by the competition between the exit demand function $O_{p}\left(n_{r p}, n_{r}\right)$, and entry supply function $I_{p}\left(n_{r p}^{\prime}, n_{r}^{\prime}\right)$ of the next region:

$$
\begin{aligned}
q_{\text {out }, r p}(t)=\min \left(O_{p}\left(n_{r p}, n_{r}\right), I_{p}\left(n_{r p}^{\prime}, n_{r}^{\prime}\right)\right), & \\
\forall p & \in \Gamma \wedge \forall r \in X
\end{aligned}
$$

The exit demand function, $O_{p}\left(n_{r p}, n_{r}\right)$, is determined as:

$$
O_{p}\left(n_{r p}, n_{r}\right)= \begin{cases}\frac{n_{r p} \delta_{r p}}{n_{r}} \frac{P_{r}\left(n_{r}\right)}{\bar{L}_{r p}} & \text { if } n_{r}<n_{c}, \\ \frac{n_{r p} \delta_{r p}}{n_{r}} \frac{P_{c}}{\bar{L}_{r p}} & \text { otherwise, }\end{cases}
$$

where $P_{r}\left(n_{r}\right)$ represents the production MFD; $P_{c}$ and $n_{c}$ represent the critical production and accumulations of region $r$, respectively.

The entry supply function of the next region $r^{\prime}$, becomes active when a congestion is observed on regional path $p$ in region $r^{\prime}$. Its formulation is [33]:

$$
I_{p}\left(n_{r p}, n_{r}\right)= \begin{cases}\frac{n_{r p} \delta_{r p}}{n_{r}} \frac{\alpha P_{c}}{\overline{L_{r p}}} & \text { if } n_{r}<n_{c}, \\ \frac{n_{r p} \delta_{r p}}{n_{r}} \frac{\alpha P_{r}\left(n_{r}\right)}{\bar{L}_{r p}} & \text { otherwise, }\end{cases}
$$

where the scaling factor $\alpha$ is set to be larger than 1 to ensure that this function is not too restrictive [33].

The inflow function $q_{i n, r^{\prime} p}(t)$ is simply the outflow function of the previous region $r^{\prime \prime}$, i.e. $q_{i n, r^{\prime} p}(t)=q_{o u t, r^{\prime \prime} p}(t)$.

This implementation of the MFD traffic model mimics the ground-truth, where one keeps track of the vehicles accumulation of each path, i.e. $n_{r p}$, and has the full information about the trip lengths $\bar{L}_{r p}$ of each path $p$ in region $r$.

\section{B. MFD traffic model for the prediction level}

Our goal is to track the vehicles' accumulations in a set of regions $r \in \Theta$, such that each of them stays close to a fixed desired set-point $n_{r}^{\text {ref }}, \forall r \in \Theta$, i.e. we target to maximize the throughput of the controlled regions. When the accumulation $n_{r}>n_{r}^{\text {ref }}, \forall r \in \Theta$, the region's throughput is reduced compared to the maximal value $n_{r}^{\text {ref }}$, leading to a suboptimal performance. In this case, we have to apply control actions to bring the accumulation of the region closer to the desired set-point, i.e. that maximizes the throughput. When the accumulation $n_{r}<n_{r}^{\text {ref }}, \forall r \in \Theta$, the region is working on free-flow conditions, and the throughput is limited by the number of vehicles that aim to exit the region. In this case, no control action is required as long as $n_{r}<n_{r}^{\text {ref }}, \forall r \in \Theta$. When the accumulation $n_{r}$ surpasses the desired set-point $n_{r}^{\text {ref }}$, the controller becomes active to prevent the system from working in sub-optimal conditions.

For the prediction model, we utilize the same exact formulation except that both $\bar{L}_{r p}$ and $n_{r p}$ should be approximated as they are unknown at this level. First, the $\bar{L}_{r p}$ is simply set to $\bar{L}_{r}$ for all paths traveling on the same region $r$. Every 
time we start a new control horizon at each $\tau$ for the NMPC, we need to estimate the initial value of $n_{r p}$ at time $t$ from the only observed variable $n_{r}$. In this paper, we use a simple approximation based on the demand traveling on all regional paths, which is defined by the path assignment:

$$
n_{r p}=\frac{\lambda_{r p}}{\sum_{p \in \Gamma} \lambda_{r p} \delta_{r p}} n_{r}, \forall p \in \Gamma \wedge \forall r \in X
$$

where $\lambda_{r p}$ is the demand traveling on regional path $p$ and region $r$; and $\delta_{r p}$ is a binary variable that equals 1 if regional path $p$ travels on region $r$.

This formulation does not account for the time lag between the flow generation at their origins and its observation in the regions. It also assumes that the demand distribution can stand for the internal flow distribution. While being a rough approximation, the simulation results will show that such an approximation is practical and effective, and a good performance level can be achieved when this approximation is embedded in the control framework.

Finally, note that the control actions will limit the flow at the region's boundaries, adding a term in Eq. 3:

$$
\begin{aligned}
q_{\text {out }, r p}(t)=\min \left(O_{p}\left(n_{r p}, n_{r}\right), I_{p}\left(n_{r p}^{\prime}, n_{r}^{\prime}\right), u_{p}^{\star}(t)\right), & \\
\forall p & \in \Gamma \wedge \forall r \in X
\end{aligned}
$$

Below we explain how to determine the gating inflows $u_{p}^{\star}(t)$ using the NMPC.

\section{Nonlinear Model Predictive Control implementation}

In this section, we describe the mathematical formulation of the NMPC to track the vehicles' accumulation in the controlled regions $r \in \Theta$. NMPC is an advanced control technique where we minimize the objective function by solving a Constrained Finite-Time Optimal Control (CFTOC) problem at each control period. The result is an optimal closed-loop gating inflow profile, where a good performance and minimal degradation are achieved over a finite time horizon. NMPC as a direct solution of the a CFTOC problem for reference matching is represented as follows:

$$
\begin{aligned}
& \left.\min _{U} \sum_{r \in \Theta} \sum_{k=0}^{N-1}\left(\| \tilde{n}_{r, k}-n_{r, k}^{\text {ref }}\right)\left\|_{Q}^{2}+\right\| \Delta u_{r, k} \|_{R}^{2}\right) \delta_{r}, \\
& \text { s.t. } \\
& n_{k+T_{s}}=f\left(n_{k}, u_{r, k}\right), \quad \forall k \in\{0, \ldots, N-1\}, \\
& \tilde{n}_{r, k}=g\left(n_{k}\right), \quad \forall k \in\{0, \ldots, N-1\}, \\
& \Delta u_{r, k}=u_{r, k}-u_{r, k-T_{s}}, \quad \forall k \in\{0, \ldots, N-1\}, \\
& u_{r, \min } \leq u_{r, k} \leq u_{r, \max }, \quad \forall k \in\{0, \ldots, N-1\}, \\
& u_{r,-1}=u_{r}\left(t-T_{s}\right), \\
& n_{0}=n(t), \\
& \forall r \in \Theta,
\end{aligned}
$$

where $U=\left\{u_{r, 0}, \ldots, u_{r, N-1}\right\}$ is a set of optimal gate inflows of all controlled regions $r \in \Theta$, for the finite-time prediction horizon $N ; u_{r}=\left\{u_{p} \delta_{r p}\right\}$ is also a set that contains the gate inflow of all regional paths that travel on the controlled region $r \in \Theta$; and $\delta_{r}$ is a binary variable that equals 1 if region $r$ is controlled, i.e. if $r \in \Theta$.

Equation (8a) is the objective function that we aim to minimize, with respect to the input flow vector $u_{r} \in \mathbb{R}^{n_{u}}$. The term $n_{u}$ represents the number of elements in vector $u_{r}$. In classical perimeter control approaches, it is considered a single value for the controller whatever the regional path is. However, in our case, we consider individual gates for each path that traveling on each of the controlled regions $r \in \Theta$. The objective function is weighted by both the output weighting matrix $Q \succeq 0$ and the input weighting matrix $R \succ 0$. The term $\tilde{n}_{r, k}$ in (8a) represents the predicted accumulation(s) for all controlled region(s) $r \in \Theta$. While, $n_{r, k}^{\text {ref }}$ represents the reference accumulation of controlled region $r$. In (8b), the state vector $n_{k+T_{s}}$ at time step $k+T_{s}$ depends on a function $f\left(n_{k}, u_{r, k}\right)$ that describes the nonlinear traffic dynamics of the network. This is the prediction model, described in the previous section. Note that the length of vector $n_{k+T_{s}}$ is equal to the total number of regions of the regional network. Both $(8 \mathrm{~g})$ - (8h) represent the initial conditions, where $u_{r}\left(t-T_{s}\right)$ and $n(t)$ are the observed traffic inputs and states in the regional network, and $T_{s}$ is the simulation/system sampling time. Equation (8e) constrains the minimum $\left(u_{r, \min }\right)$ and maximum $\left(u_{r, \max }\right)$ inflows of the paths traveling on the controlled region(s) $r$. Equation (8c) represents the estimated output accumulation.

Fig. 2 (a) depicts a schematic representation of how the plant and prediction models interact together. In the plant model, the traffic dynamics are simulated through an accumulation-based MFD model as described in the previous section, where the trip lengths $\bar{L}_{r p}$ in (4) and (5) are calculated by the reference level $M_{r}$, i.e. the related regional path $p$. The first time instants correspond to the network loading (or warmup period). Once the network is loaded, the NMPC controller gets activated when the accumulation(s) in the controlled region(s) reach the set-point(s). We then run the prediction model over the finite-time prediction horizon $N$ to determine the optimal inflow gates $u_{p}$. The optimal control actions are then taken into account in the plant model. We only apply the first optimized gating inflows $u_{r, 0}^{\star}$ to the plant. This procedure is also known as the Receding Horizon Controller (RHC) as discussed in [22], [23], [25]. It is worth to mention that we measure plant states at each simulation sampling time $T_{s}$ whereas the MPC is employed at each controller sampling time $\tau$ and the same value is applied to plant until next $\tau$. Here, $T_{s} \leq \tau$ is considered to show the effectiveness of the proposed approach in real-life.

One of the bottlenecks in NMPC solution is the extensive optimization and the resulting computational requirements. The optimization problem described by the system of equations $(8 \mathrm{a})-(8 \mathrm{~g})$ is nonlinear and non-convex. Thus a proper selection of an optimization solver has to be made in order to obtain feasible optimal control values. There are several nonlinear programming algorithms that can be applied to solve this optimization problem, such as the Interior Point Methods (IPM), the Active Set Method (ASM), or the Sequential 
Quadratic Programming (SQP) (see e.g. [39], [40]). In this paper, we choose the SQP method as it is strictly feasible with respect to constraints, robust, and has a better warm-starting properties than IPM and ASM [41].
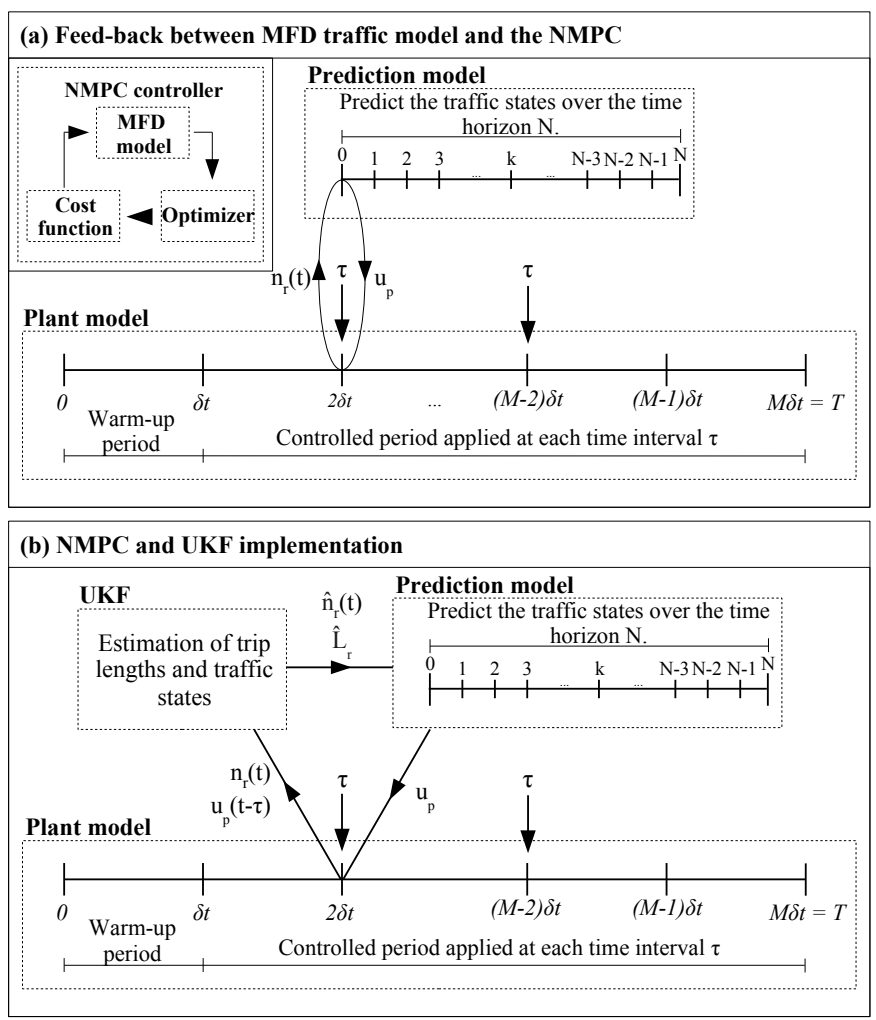

Fig. 2: (a) Schematic implementation of the NMPC together with the MFD traffic model, i.e. the plant. (b) Inclusion of the UKF in the framework depicted in (a), to correct for the bad estimations of the trip lengths $\tilde{L}_{r}$.

\section{Combined State-Parameter Estimation using Unscented Kalman Filter}

In reality, the average trip length $\bar{L}_{r}$ changes over time [10]. One possibility to account for the time-dependence of $\bar{L}_{r}$ is to consider (1). But, as discussed in the Introduction, $\bar{L}_{r p}$ is challenging to calibrate, and $n_{r p}(t)$ is impossible to measure in reality. We then propose a methodological framework to dynamically adjust $\bar{L}_{r}$. We make use of a combined strategy based on the application of an UKF [42], [43] and NMPC (see Fig. 2 (b)).

The Kalman Filter (KF) is a widely used conceptual twostages prediction-correction approach. The KF fundamental operation is a successive process of prediction based on system input, followed by a correction process based on measurable system output. The Extended Kalman Filter (EKF) is the most commonly used technique in the field of nonlinear system estimation. This is mainly due to the simplicity of its implementation. The EKF has been applied in many studies in the literature for traffic states or parameter estimation (see, e.g. [44]-[46]). The EKF approximates the existent system non-linearities, using the Jacobian propagation of co-variance information. This leads to the loss of higher order information, and the precision of both states estimation and error covariance are degraded. The UKF offers an appealing alternative for dynamically adjust the trip lengths (i.e. parameter estimation) and accumulations (i.e. state estimation), without the need to linearize the system (see e.g. [42], [47]). Using the UKF, we adjust the average trip lengths $\tilde{L}_{r}, \forall r \in X$, as well as the initial traffic conditions $n_{r, 0}$. Both $\tilde{L}_{r}$ and $n_{r, 0}$ will then be set as inputs to the used in the prediction model, i.e. the NMPC. Below, we describe in more detail the UKF implementation.

Fig. 2 (b) depicts a schematic representation of how the NMPC is applied together with the UKF. The process is simple. The UKF receives as an input the observed traffic states, i.e. measured accumulations $n_{r}(t), \forall r \in X$, and the gating inflows $u_{p}^{\star}(t)$ of the previous control action. The UKF is used for parameter estimation (trip lengths, i.e. $\vec{L}=$ $\left\{\bar{L}_{r}\right\}, \forall r \in X$ ) along with state estimation (accumulations, i.e. $\left.\hat{n}_{r}(t), \forall r \in X\right)$ for all regions of the network. The goal is to find the optimal set of trip lengths $\vec{L}$, such that the difference between the estimated $\hat{n}_{r}(t), \forall r \in X$ and measured accumulations $n_{r}(t), \forall r \in X$ is minimized. For this purpose, we add the parameter vector $\left(\tilde{L}_{r, k}\right)$ into the state vector $\left(n_{k}\right)$ and define the new state update equation accordingly. In practise, we add the time-varying parameters to the state vector as $\tilde{L}_{r, k+\delta t}=\tilde{L}_{r, k}, \forall r \in X$. We define $n_{e, k}=\left[\begin{array}{ll}n_{k}^{T} & \tilde{L}_{r, k}^{T}\end{array}\right]^{T}$ as the augmented state vector, and $\hat{n}_{e, k}=\left[\begin{array}{ll}\hat{n}_{k}^{T} & \tilde{L}_{r, k}^{T}\end{array}\right]^{T}$ as its estimation. The UKF estimation method considers $2 \eta_{n, e}+1$ sigma points sampled from the initial confidence interval. These points are propagated in time using the system model. Note that, $\eta_{n, e}=\eta_{n}+\eta_{p}$ is the number of augmented states in the system, where $\eta_{p}$ and $\eta_{n}$ represent the number of parameters and states, respectively. In this paper, we follow the UKF formulation as described in [42], [43]. The nonlinear system is:

$$
\begin{aligned}
n_{e, k+T_{s}} & =f\left(n_{k}, \tilde{L}_{r p, k}, u_{r, k}\right)+w_{k}, \\
y_{e, k} & =g\left(x_{k}\right)+v_{k},
\end{aligned}
$$

where $w_{k}$ and $v_{k}$ represent the process and output noise vectors, respectively. Note that $w_{k}$ and $v_{k}$ have zero mean values, and co-variance matrices $\bar{Q}_{k}$ and $\bar{R}_{k}$, respectively.

The UKF computes the current estimate of $\hat{n}_{e, k}$, by solving the MFD model described in (9a). The current state estimates $\hat{n}_{e, k}$ and output estimation $y_{e, k}$ are updated at time $k$ as:

$$
\begin{aligned}
& \hat{x}_{e, k}=\hat{x}_{e, k-1}+\kappa\left(y_{e, k}-\hat{y}_{e, k}\right), \\
& \hat{y}_{e, k}=\hat{x}_{e, k},
\end{aligned}
$$

where $\hat{n}_{e, k-1}$ and $\hat{n}_{e, k}$ are the vectors of apriori and posteriori state estimates, respectively. The term $\kappa$ represents the Kalman gain.

The detailed system of equations and solution algorithm are presented in [42], [48].

The output of the UKF consists of the calibrated average trip lengths $\vec{L}=\left\{\bar{L}_{r}\right\}, \forall r \in X$ and estimated traffic states $\hat{n}_{k}$. We then set $\hat{L}_{r}=\tilde{L}_{r}$ for running the prediction model. The estimated accumulations are also set as the initial condition for the prediction model, i.e. $n_{0}=\hat{n}_{k}$. The prediction model 
is then solved as described in the previous section, for one sample time $k$. We apply control actions with the properly adjusted trip lengths at each $\tau$ seconds and until the $T$ is reached.

\section{E. Setting of the different controller configurations}

In this paper, we set-up four different configurations of the model-based perimeter control strategy. In all scenarios, the dynamics of the plant model are represented by an accumulation-based MFD model where the trip lengths are calibrated according to the reference level $M_{r}$, i.e. $\bar{L}_{r p}$. The main difference between the four scenarios lies on how the trip lengths are calibrated for the prediction model:

- Scenario 1: Uncontrolled. In this scenario, we do not apply any kind of optimal control actions on the plant model.

- Scenario 2: Perfect model-based controller. In this case, we simply consider that the prediction model is the plant model, disregarding any calibration and monitoring issues. While being unrealistic in practise, this scenario provides an optimal case for the controller's efficiency. In this scenario, we have perfect knowledge of the trip lengths $\left(\bar{L}_{r p}\right)$ and accumulations $\left(n_{r p}\right)$ per path.

- Scenario 3: Regular model-based controller. We use the regular prediction model as described in Sect. II-C. In this scenario, we consider static average trip lengths per region, $\bar{L}_{r}$, independent of the path that vehicles are traveling. In terms of the accumulations, at the prediction level, we are only able to estimate accumulations per region, $n_{r}$, from the plant model. We then estimate the accumulations per region using Eq. 6.

- Scenario 4: Regular model-based controller with UKF, to update accumulations. We assume the same settings as in the previous Scenario 3. The difference is that we utilize the UKF to predict the input accumulations $\hat{n}_{r}, \forall r \in X$ to the prediction model. At each control period $\tau$, we dynamically estimate the input accumulations using the proposed UKF model.

- Scenario 5: Regular model-based controller with UKF, to update accumulations and trip lengths. We assume the same settings as in the previous scenario 4 . However, we also determine the average trip lengths $\bar{L}_{r}$ as function of the changes in the traffic conditions in the regions. At each control period $\tau$, we dynamically estimate the dynamic average trip lengths as well as the accumulations, using the proposed UKF model. Our goal is to show that the dynamically adjusting trip lengths and accumulations improve the controller's performance. For this, we chose a simpler formulation without considering a time-lag between the prediction and the plant models. This means that we estimate the accumulations and trip lengths at the same time step. This simpler implementation still proves our case.

\section{RESULTS}

\section{A. Definition of the test scenarios}

The test network is depicted in Figure 3 (a) and encompasses the $3^{\text {rd }}$ and $6^{\text {th }}$ districts of Lyon and the city of Villeur- banne (France). We refer to this network as L63V. It has 3127 nodes and 3363 links, and is partitioned into seven regions. Figure 3 (b) shows the MFD functions for these regions. They were calculated by assuming a bi-parabolic shape to fit microscopic simulation data obtained from Symuvia [49].
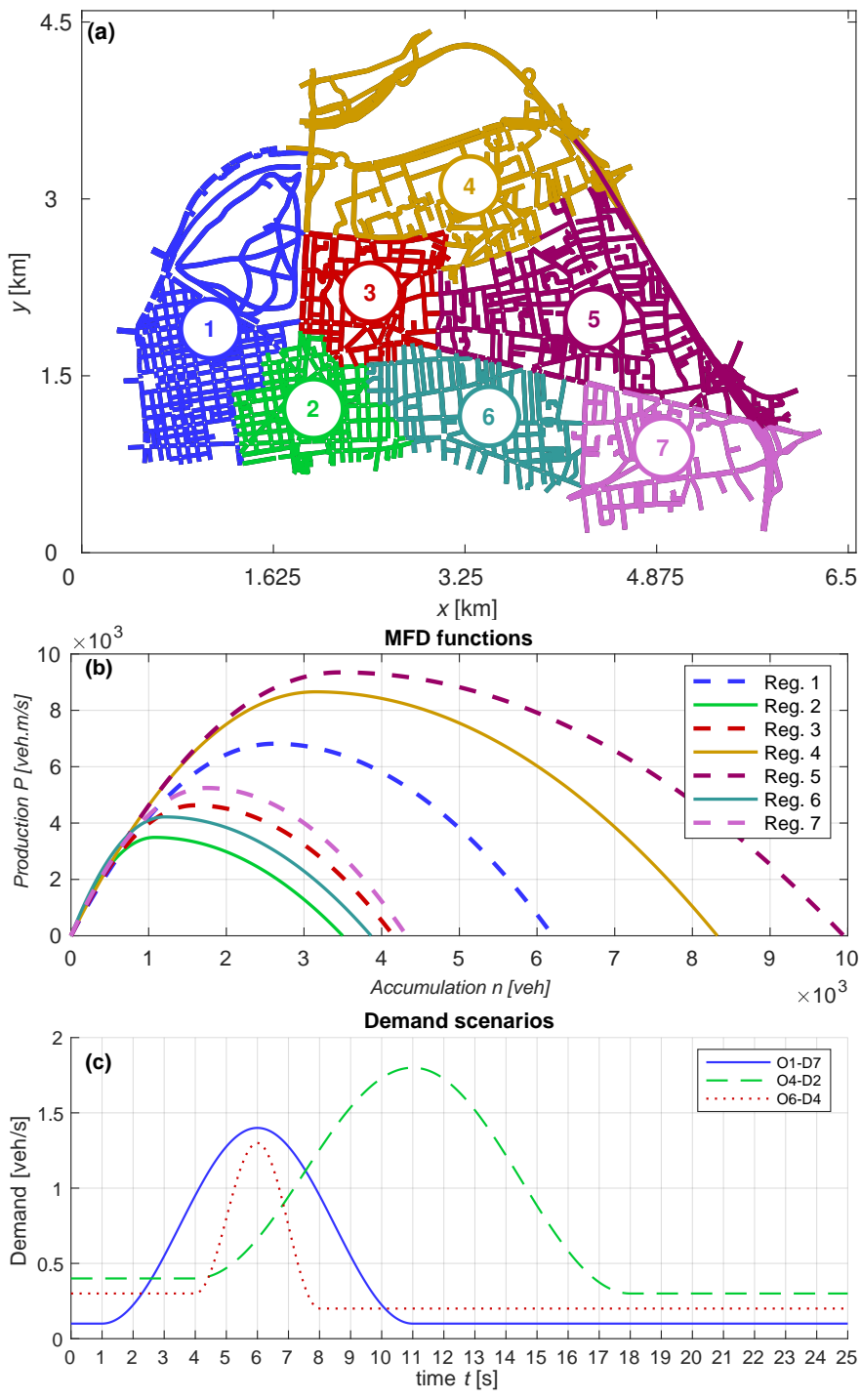

Fig. 3: (a) City network composed by the $3^{\text {rd }}$ and $6^{\text {th }}$ districts of Lyon and Villeurbanne (France), divided into seven regions. (b) MFD functions. (c) Demand scenarios.

The regional paths and trip lengths are determined based on a set of virtual trips in the city network. We sample a total of 3 million origin and destination nodes on the L63V network (Figure 3 (a)), and calculate the shortest-trip in distance between each of them. We then scale-up these trips according to the definition of the city network partitioning depicted in Figure 3 (a). The regional paths are ranked according to their significance level. In this test case, we consider 3 regional OD pairs: $1-7 ; 4-2$; and $6-4$. The regional choice sets are: $\Omega^{17}=\{1267,1357\} ; \Omega^{42}=\{412,432\}$; and $\Omega^{64}=\{654,6534\}$. Let $\Gamma$ define the set containing these five regional paths. We calculate the average trip lengths $\bar{L}_{r}$ and $\bar{L}_{r p}$ for these regional paths (see also [8] for the mathematical 
details). Figure 3 (c) depicts the demand scenarios for the three OD pairs. We assign $80 \%$ of the OD 17 demand to path $p=\{1357\}$. While, $85 \%$ of the OD 42 demand is assigned to $p=\{432\}$. The whole demand of OD 64 is assigned to path $p=\{6534\}$. For both plant and prediction MFD traffic models, we fix a total simulation period of $T=25000$ seconds, and the traffic states are determined at every $\delta t=10$ seconds in the regions.

In this paper, we target to control the total accumulation in region 3 to a maximum of 700 vehicles, which corresponds to the maximum outflow. This means that the set $\Theta$ only includes region 3, and the reference accumulation $n_{3, k}^{r e f}=700$ vehicles. There are three paths that cross region 3 . We then have to define three overall gates (with one or more intersections each) at the perimeter of region 3 and therefore $n_{u}=3$. We set $u_{3, \max }$ based on the maximum inflows verified for the scenario where no control is applied to region 3. The sets of values used for these constraints are: $[0.1,0.1,0.1] \leq u_{3, k} \leq[1.25,1.70,0.95]$. We also set $Q=0.1 \times \mathbb{I}_{(3 \times 3)}$ and $R=1 \times \mathbb{I}_{\left(n_{y} \times n_{y}\right)}$, where $n_{y}$ corresponds to the number of elements of the output vector. In our case, we are just estimating the accumulation in region 3 , i.e. $\tilde{n}_{3, k}$, and then $n_{y}=1$. Note that, for calibrating the $Q$ and $R$ parameters, we run different simulations with different sets of $Q$ and $R$ values for the previously described demand scenario, and check their performance in region 3 . We then choose the set of values that yield the best performance, see [50, Chapter 7] for tuning the MPC. The total number of sample times $N$ is a user pre-defined parameter, that should be set respecting the fact that the prediction horizon (i.e. $N \times \tau$ ) is much larger than the time difference between two control actions. We then set $N=10$. For the implementation of the UKF, we set the process noise vector as $w=\left[\begin{array}{lll}10^{-6} \times \mathbb{I}_{(7 \times 7)} & 10^{-2} \times \mathbb{I}_{(7 \times 7)}\end{array}\right]^{T}$; the measurement noise vector as $v=10^{-6} \times \mathbb{I}_{\left(n_{y} \times n_{y}\right)}$, where $n_{y}=1$ because we are just optimizing the accumulation of region 3 ; and the state co-variance vector as $P=\left[\begin{array}{ll}1 \times \mathbb{I}_{(7 \times 7)} & 10 \times \mathbb{I}_{(7 \times 7)}\end{array}\right]^{T}$. We also set to 1 , both the spread of sigma points and the characterization of the state distribution used to adjust the weights of the transformed sigma points.

\section{B. Influence of trip length approximation on the prediction model}

In this section, we show: (i) how the assumption of static average trip lengths per region $\bar{L}_{r}$ degrades the controller's performance, for lower but more realistic $\tau$ values (i.e. scenario 3); and (ii) that the use of the UKF to dynamically estimate the average trip lengths and accumulations improves the controller's performance (i.e. scenario 5). The controller's performance is evaluated against the reference scenario 2, where we assume to have the full knowledge about the average trip lengths and accumulations per path, for the prediction model. We recall that scenario 2 is just an hypothetical and idealized scenario, since in reality we can only estimate both accumulations $n_{r}$ and trip lengths $\bar{L}_{r}$ per region. We consider this ideal scenario only for the purposes of discussion in this section, and evaluate the controller's performance of scenarios 3,4 and 5 in comparison to this one.
In the test case described in the previous section, our goal is to maximize the throughput of region 3 . We apply optimal control actions in region 3, at each $\tau=10,60$, and 120 seconds. The larger values of $\tau$ are more realistic as the cycle length of traffic signals is usually $\sim 60$ seconds. We then discuss how to implement the UKF for adjusting the average trip lengths for the prediction model.

Fig. 4 (a)-(c) depicts the evolution of the accumulation $n_{3}(t)$ in region 3, during the controlled period. The results are shown for the five scenarios considered in this study, and the three values of $\tau$. Fig. 4 (d) depicts the total time spent (TTS) by all drivers in region 3 for the same previous scenarios 2 to 5. The TTS for the uncontrolled scenario 1 is $4.295 \times 10^{5}$ seconds. Fig. 6 shows the evolution of the gating inflows $u_{p}(t)$ as function of the simulation time, for the three paths (i.e. $p=\{1357\}, p=\{432\}$ and $p=\{6534\})$ and the different $\tau$ values.
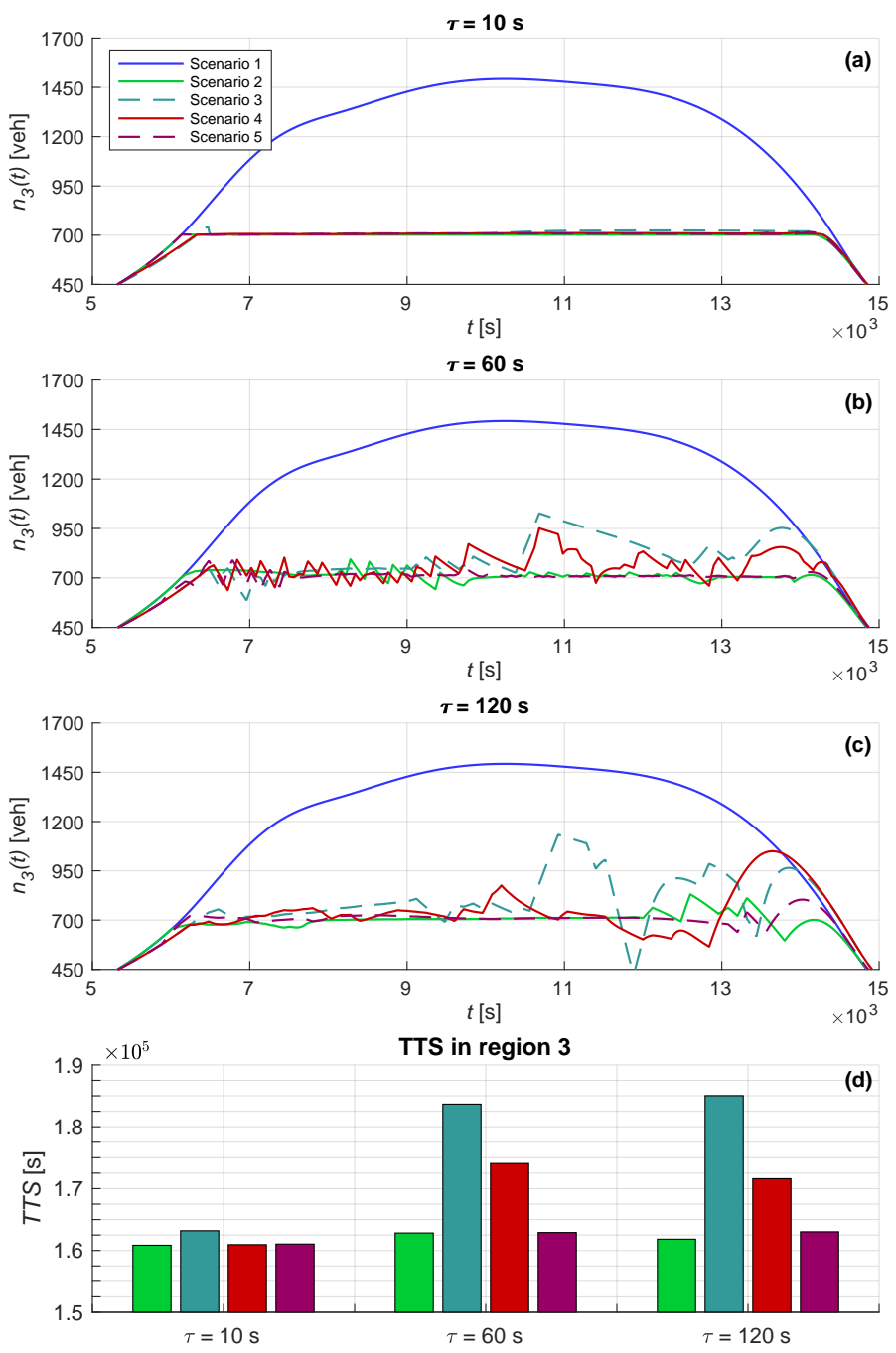

Fig. 4: (a)-(c) Evolution of the traffic dynamics in the controlled region 3, for all five scenarios. The results are depicted for: (a) $\tau=10 \mathrm{~s}$; (b) $\tau=60 \mathrm{~s}$; and (c) $\tau=120 \mathrm{~s}$. (d) Total time spent (TTS) in region 3 for scenarios 2 to 5, and for all three values of $\tau$. 

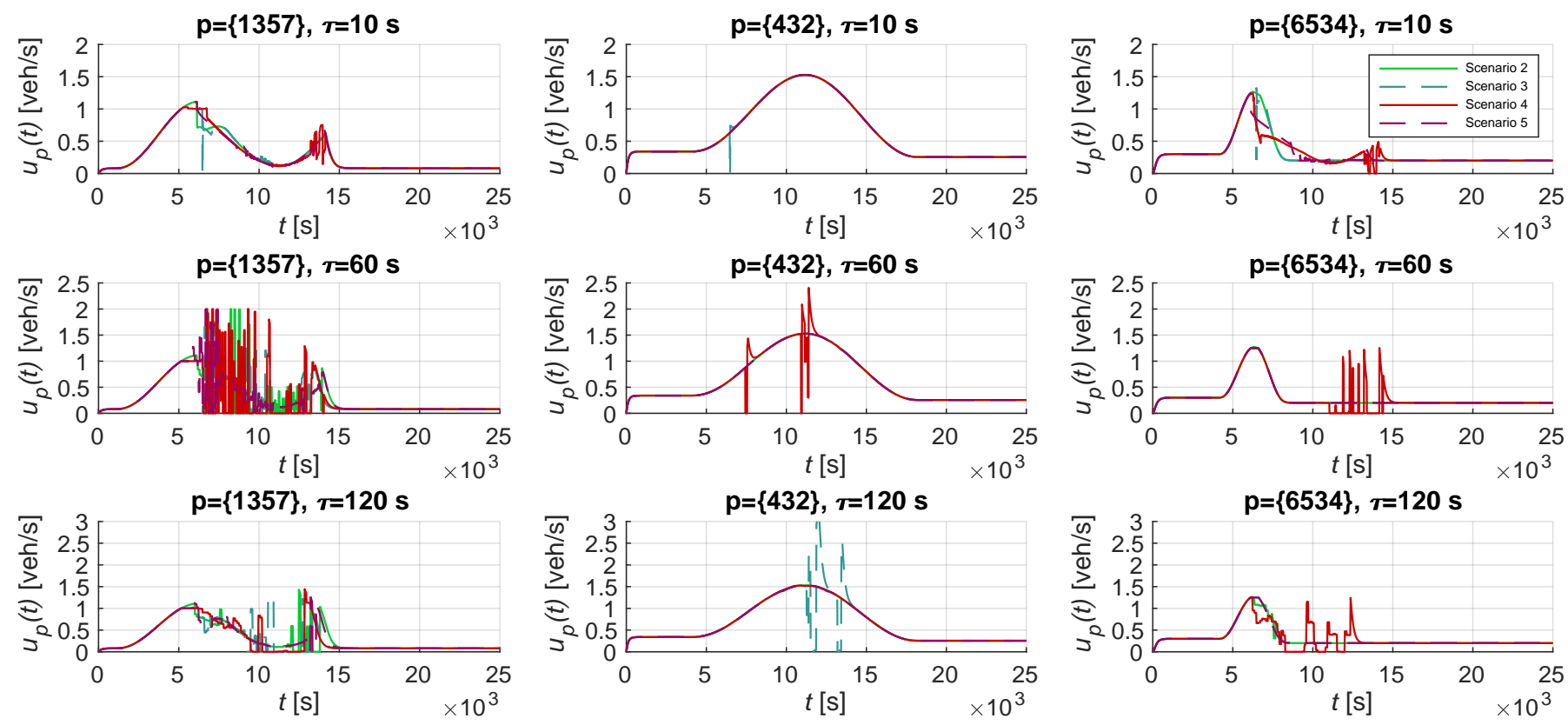

Fig. 5: Gating inflows $u_{p}(t)$ as function of the simulation time $t$ [s], for the controlled region 3 . The different panels depict the results for the three regional paths $p$ that travel region 3, and for the different $\tau=10,60,120$ seconds.
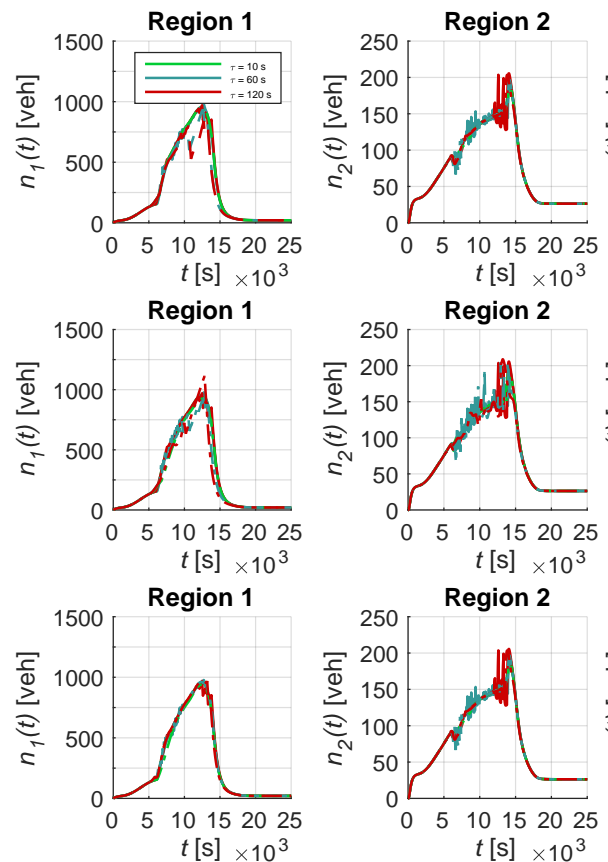
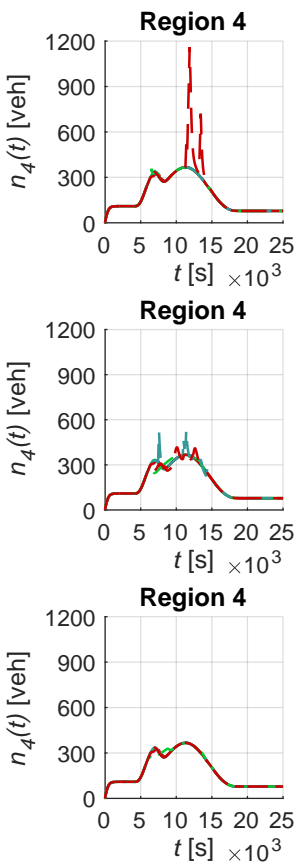
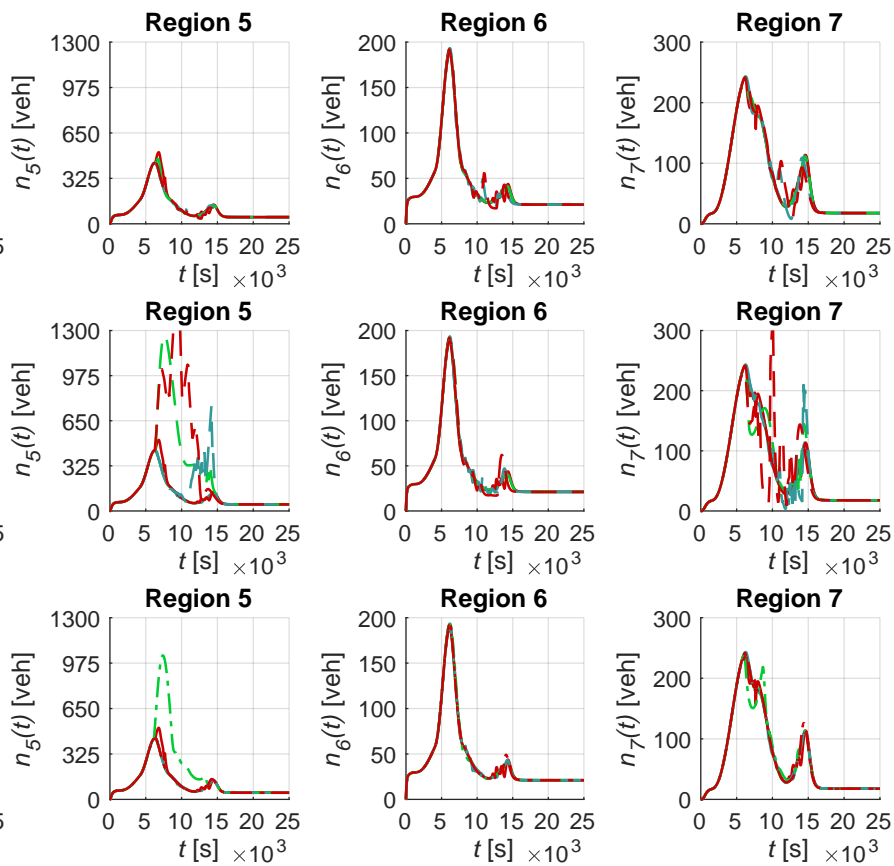

Fig. 6: Same as in Fig. 4 (a)-(c), but for the non controlled regions. The plots represent the comparison between the Scenarios 3 (top row panels), Scenario 4 (middle row panels) and Scenario 5 (bottom row panels) against the benchmark Scenario 2.

One can observe in Fig. 4 (a) that the accumulation $n_{3}(t)$, for scenario 3 , is kept close to the one of the optimal scenario 2. This shows that for high frequency updates of the optimal control actions, i.e. $\tau=10$ seconds, the system is able to adapt itself to the static assumption of average trip lengths used in the prediction model. However, the differences between the accumulations $n_{3}(t)$ determined for scenarios 3 and 2, increase as the frequency of the optimal control actions decreases. We can observe that in Fig. 4 (b) and (c), the controller is able to maintain the region's accumulation close to 700 vehicles, for the reference scenario 2. In contrast, for scenario 3, the region becomes congested after approximately 10000 seconds. The controller is not being able to maintain the region's accumulation close to the desired value of 700 vehicles, i.e. the throughput of the region is reduced and leads to a suboptimal performance as also evidenced by the increase of the TTS (see Fig. 4 (d)). This happens because the controller is not able to properly regulate the gating inflow of vehicles 
traveling on path $p=\{1357\}$ entering region 3 , as it can be observed in Fig. 6 for scenario 3. These results clearly show that the assumption of static average trip lengths degrades the controller performance compared to the optimal scenario 2. The differences become more evident as the frequency of the optimal control actions decreases. The relative differences between the TTS of scenario 3 and 2, are 1.5\%, $12.8 \%$ and $14.3 \%$, for for $\tau=10,60$ and 120 seconds, respectively. This means that, for example, for $\tau=120$ seconds, the TTS of scenario 3 is $14.3 \%$ larger than the TTS of scenario 2 .

The application of the UKF proves to be effective to adjust the average trip lengths $\bar{L}_{r}$ according to the changes of the traffic conditions in the regions as well as the accumulations $n_{r}$. As one can observe in Fig. 4, the controller is able to maintain the accumulation $n_{3}(t)$ determined for scenario 5, close to the one of the reference scenario 2. While, this is not true for scenario 4, where we only adjust the accumulations. For this scenario 4 and for $\tau=120$ seconds, we observe that in the simulation time between 12000 and 13000 seconds, the accumulation $n_{3}(t)$ is inferior to the reference value. This happens because we do not linearize the control optimization. Instead, we solve directly the non-linear optimization problem, and the solver might find sub-optimal solutions.

The relative differences in the TTS by drivers in region 3 is reduced for scenario 4 , however it is still larger than the reference scenario 2 . The situation is clearly improved for scenario 5, where the TTS is close to the one of scenario 2 . We note that the TTS of scenario 5 is larger than the TTS of scenario 2, for all three values of $\tau$. The relative differences between the TTS of: (i) scenarios 4 and 2, are $0.1 \%, 6.9 \%$ and $6.0 \%$, for $\tau=10,60$ and 120 seconds, respectively; and (ii) scenarios 5 and 2, are $0.1 \%, 0.1 \%$ and $0.7 \%$, for the same ordering of the $\tau$ values. This clearly shows that the improvement on the controller's performance comes from adjusting both trip lengths and accumulations. Therefore, these results validate the application of the UKF to properly adjust the input parameters, i.e. $\bar{L}_{r}$ and $n_{r}$, used to run the prediction model, for more realistic $\tau$ values.

The results depicted in Fig. 4 also show that the way we estimate the accumulations per region using Eq. 6 in the prediction model, is effective. We recall that this approach is used for all scenarios 3, 4 and 5. If this assumption was not effective, we would not observe a close performance of the controller in maximizing the throughput of region 3 , when comparing scenarios 5 and 2.

Fig. 6 shows the evolution of the traffic dynamics in the non controlled regions of the network. The application of the UKF also improves the estimation of the congestion propagation in the whole network, that is verified by the close evolution trends between scenarios 2 and 5, for all three $\tau$ values. This effect is particularly observed in the adjacent region 1 , where the assumption of static trip lengths leads to an underestimation of the congestion with respect to the case of the perfect modelbased controller, i.e. scenario 2. However, the application of the UKF corrects for this bias.

We also analyze the UKF estimation results of the trip lengths over the simulation time. For this, we determine the relative differences $\left(\Phi_{r}(t)\right)$ between the estimated trip
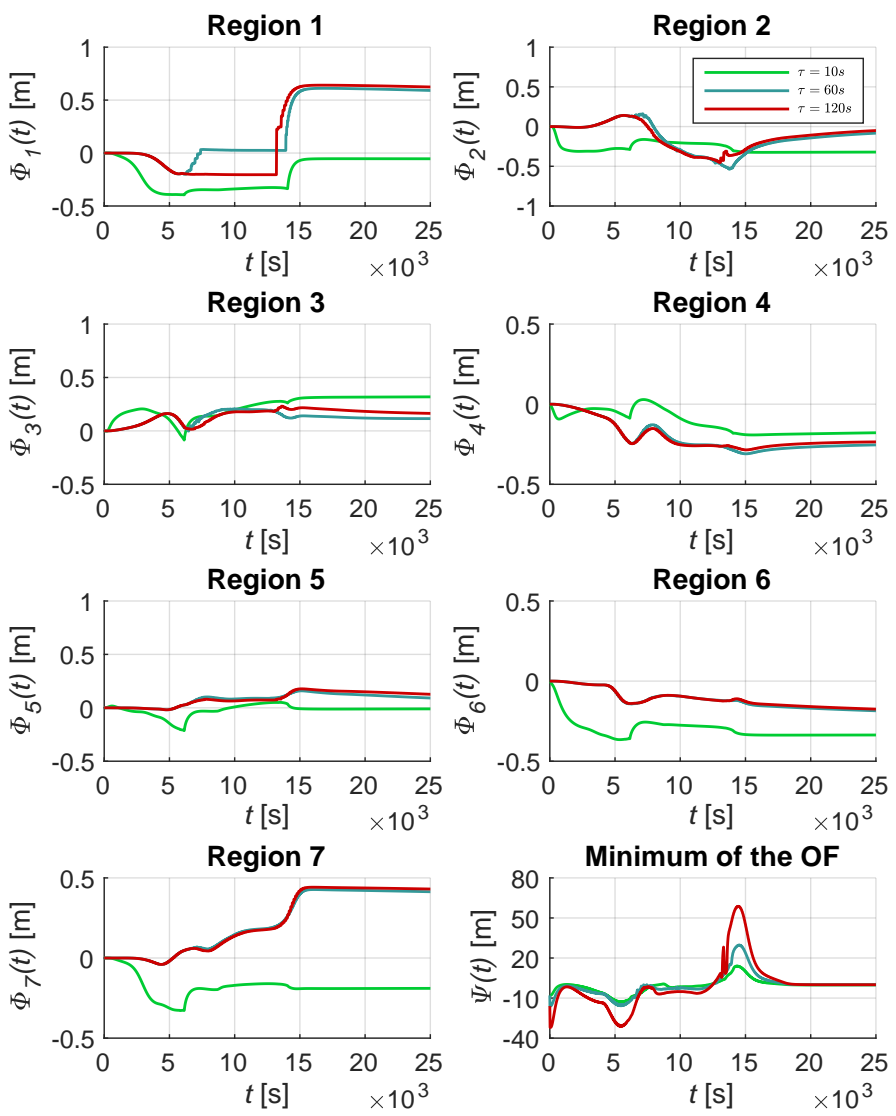

Fig. 7: Relative differences $\Phi_{r}(t)$ for all seven regions of the network. The minimum of the objective function is also depicted.

lengths $\left(\hat{L}_{r}\right)$ through the UKF (scenario 5) and the static average trip lengths $\left(\bar{L}_{r}\right)$ calculated through $M_{s}$ (scenario $3)$. The relative differences for a region $r$ are calculated as $\Phi_{r}(t)=\frac{\hat{L}_{r}(t)-\bar{L}_{r}}{\bar{L}_{r}}, \forall r \in X$. We also determine the value of the objective function $\Psi(t)$, based on the differences between the estimated accumulations $\hat{n}_{r}(t)$ and the observed accumulations $n_{r}(t)$, as $\Psi(t)=\sum_{r \in X} \hat{n}_{r}(t)-n_{r}(t)$. Fig. 7 depicts the evolution of the relative differences $\Phi_{r}(t)$ for all seven regions as well as the three values of $\tau$ considered. This figure also shows the temporal evolution of the minimum of the objective function $\Psi(t)$.

One can observe that for $\tau=10$ seconds, the relative errors are in general small because the system is able to adapt itself to the static trip lengths due to the large frequency updates of the optimal control actions. But, the relative differences increase for larger values of $\tau$. This is consistent with the improvement provided by the UKF in the controller's performance, and prediction of the system dynamics in the system, as previously discussed. The objective function $\Psi(t)$ is in general close to 0 . However, this is not observed during the beginning (i.e. around $\sim 5000$ seconds) and ending (i.e. around $\sim 15000$ seconds) of the controlled period in region 3 . This is also consistent with the matching differences verified in Fig. 4 (a)-(c), between the scenarios 2 and 5 , during these two periods. 


\section{CONCLUSION}

In this paper, we show that: (i) the assumption of static average trip lengths $\bar{L}_{r}$ clearly degrades the performance of the controller with respect to the reference scenario 2 , and the throughput of the region is reduced leading to a suboptimal performance level; and (ii) the proposed methodology based on the UKF is effective for improving the controller's performance. With the UKF, the controller is able to keep the accumulation $n_{3}(t)$ close to both the desired set-point and the accumulation determined for the reference scenario 2. This fact is also illustrated by the total time spent (TTS) in region 3 , for scenarios 5 and 2. The TTS of these two scenarios are very close, however the TTS of scenario 5 is slightly superior. Note that, we do not expect that the UKF tracks the exact value of the average trip lengths $\bar{L}_{r}$, because the prediction and plant models have different structures. Even if we calibrate $\bar{L}_{r}$ at the end of the control period, it means that the trip lengths are the same for all regional paths traveling on the same region, which is known to introduce significant bias in the predicted traffic states [8]. It is important to stress here that the UKF provides good estimations of the average trip lengths $\bar{L}_{r}$ and accumulations $n_{r}$ per region, which are utilized as inputs in the prediction model, to improve the performance of the controller. The performance achieved by the controller in this scenario is very close to the one achieved in the case when the prediction perfectly fits the plant model, i.e. scenario 2 . This is highly important for control applications in practise. The results show an improvement in the performance of the controller in region 3, bringing it close to the best performance that can be achieved with a perfect prediction model. Moreover, the estimation of the traffic dynamics in the whole system is also improved, and closer to the reference scenario.

As future research work, we aim to extend the proposed methodological framework to incorporate a route guidance system [51].

\section{ACKNOWLEDGMENT}

The authors thank all comments and suggestions, from the two anonymous reviewers, that have much improved our paper. S. F. A. Batista and M. Menéndez acknowledge support by the NYUAD Center for Interacting Urban Networks (CITIES), funded by Tamkeen under the NYUAD Research Institute Award CG001 and by the Swiss Re Institute under the Quantum Cities ${ }^{\mathrm{TM}}$ initiative. D. Ingole, and L. Leclercq acknowledge funding by the European Research Council (ERC) under the European Unions Horizon 2020 research and innovation program (grant agreement No 646592 - MAGnUM project).

\section{REFERENCES}

[1] C. Daganzo, "Urban gridlock: Macroscopic modeling and mitigation approaches," Transportation Research Part B: Methodological, vol. 41, pp. 49-62, 2007.

[2] N. Geroliminis and C. Daganzo, "Existence of urban-scale macroscopic fundamental diagrams: Some experimental findings," Transportation Research Part B: Methodological, vol. 42, pp. 759-770, 2008.

[3] M. Saeedmanesh and N. Geroliminis, "Clustering of heterogeneous networks with directional flows based on "snake" similarities," Transportation Research Part B: Methodological, vol. 91, pp. 250-269, 2016.
[4] C. Lopez, L. Leclercq, P. Krishnakumari, N. Chiabaut, and H. van Lint, "Revealing the day-to-day regularity of urban congestion patterns with 3d speed maps,' Scientific Reports, vol. 7, pp. 1-11, 2017.

[5] M. Saeedmanesh and N. Geroliminis, "Dynamic clustering and propagation of congestion in heterogeneously congested urban traffic networks," Transportation Research Part B: Methodological, vol. 105, pp. 193-211, 2017.

[6] G. Casadei, V. Bertrand, B. Gouin, and C. Canudas-de-Wit, "Aggregation and travel time calculation over large scale traffic networks: An empiric study on the grenoble city," Transportation Research Part C: Emerging Technologies, vol. 95, pp. 713-730, 2018.

[7] L. Ambühl, A. Loder, N. Zheng, K. W. Axhausen, and M. Menendez, "Approximative network partitioning for mfds from stationary sensor data," Transportation Research Record, vol. 2673, 2019.

[8] S. F. A. Batista, L. Leclercq, and N. Geroliminis, "Estimation of regional trip length distributions for the calibration of the aggregated network traffic models," Transportation Research Part B: Methodological, vol. 122, pp. 192-217, 2019.

[9] M. Ramezani, J. Haddad, and N. Geroliminis, "Dynamics of heterogeneity in urban networks: aggregated traffic modeling and hierarchical control," Transportation Research Part B, vol. 74, pp. 1-19, 2015.

[10] M. Yildirimoglu, I. I. Sirmatel, and N. Geroliminis, "Hierarchical control of heterogeneous large-scale urban road networks via path assignment and regional route guidance," Transportation Research Part B: Methodological, vol. 118, pp. 106-123, 2018.

[11] I. I. Sirmatel and N. Geroliminis, "Economic model predictive control of large-scale urban road networks via perimeter control and regional route guidance," IEEE Transactions on Intelligent Transportation Systems, vol. 19, pp. 1112-1121, 2018.

[12] K. Yang, N. Zheng, and M. Menendez, "Multi-scale perimeter control approach in a connected-vehicle environment," Transportation Research Part C: Emerging Technologies, vol. 94, pp. 32-49, 2018.

[13] K. Yang, M. Menendez, and N. Zheng, "Heterogeneity aware urban traffic control in a connected vehicle environment: A joint framework for congestion pricing and perimeter control," Transportation Research Part C: Emerging Technologies, vol. 105, pp. 439 - 455, 2019.

[14] D. Ingole, G. Mariotte, and L. Leclercq, "Minimizing network-wide emissions by optimal routing through inner-city gating," Transportation Research Part D: Transport and Environment, vol. 86, p. 102411, 2020.

[15] Y. Ren, Z. Hou, I. I. Sirmatel, and N. Geroliminis, "Data driven model free adaptive iterative learning perimeter control for large-scale urban road networks," Transportation Research Part C: Emerging Technologies, vol. 115, p. 102618, 2020.

[16] J. Haddad and N. Geroliminis, "On the stability of traffic perimeter control in two-region urban cities," Transportation Research Part B: Methodological, vol. 46, p. 11591176, 2012.

[17] N. Geroliminis, J. Haddad, and M. Ramezani, "Optimal perimeter control for two urban regions with macroscopic fundamental diagrams: a model predictive approach," IEEE Transactions on Intelligent Transportation Systems, vol. 14, pp. 348-359, 2013.

[18] A. Kouvelas, M. Saeedmanesh, and N. Geroliminis, "A linear formulation for model predictive perimeter traffic control in cities," IFACPapersOnLine, vol. 50, no. 1, pp. 8543-8548, 2017.

[19] J. Haddad, M. Ramezani, and N. Geroliminis, "Cooperative traffic control of a mixed network with two urban regions and a freeway," Transportation Research Part B: Methodological, vol. 54, pp. 17 - 36, 2013.

[20] S. Lin, B. De Schutter, Y. Xi, and H. Hellendoorn, "Fast model predictive control for urban road networks via milp," IEEE Transactions on Intelligent Transportation Systems, vol. 12, no. 3, pp. 846-856, 2011.

[21] H. Fu, N. Liu, and G. Hu, "Hierarchical perimeter control with guaranteed stability for dynamically coupled heterogeneous urban traffic," Transportation Research Part C: Emerging Technologies, vol. 83, pp. 18-38, 2017.

[22] D. Q. Mayne, "Model predictive control: Recent developments and future promise," Automatica, vol. 50, no. 12, pp. 2967-2986, 2014

[23] L. Grüne and J. Pannek, Nonlinear Model Predictive Control : Theory and Algorithms. 2nd Edition, ser. Communications and Control Engineering. Cham, Switzerland: Springer, 2017.

[24] J. A. Rossiter, Model-based predictive control: a practical approach. CRC press, 2017.

[25] F. Borrelli, A. Bemporad, and M. Morari, Predictive control for linear and hybrid systems. Cambridge University Press, 2017.

[26] J. Haddad and A. Shraiber, "Robust perimeter control design for an urban region," Transportation Research Part B: Methodological, vol. 68, pp. 315-332, 2014. 
[27] M. Hajiahmadi, J. Haddad, B. De Schutter, and N. Geroliminis, "Optimal hybrid perimeter and switching plans control for urban traffic networks," IEEE Transactions on Control Systems Technology, vol. 23, no. 2, pp. 464-478, 2015.

[28] I. I. Sirmatel and N. Geroliminis, "Nonlinear moving horizon estimation for large-scale urban road networks," IEEE Transactions on Intelligent Transportation Systems, pp. 1-12, 2019.

[29] K. Aboudolas and N. Geroliminis, "Perimeter and boundary flow control in multi-reservoir heterogeneous networks," Transportation Research Part B: Methodological, vol. 55, pp. 265-281, 2013.

[30] J. Haddad, "Optimal perimeter control synthesis for two urban regions with aggregate boundary queue dynamics," Transportation Research Part B: Methodological, vol. 96, pp. 1-25, 2017.

[31] R. Zhong, C. Chen, Y. Huang, A. Sumalee, W. Lam, and D. Xu, "Robust perimeter control for two urban regions with macroscopic fundamental diagrams: A control-lyapunov function approach," Transportation Research Part B: Methodological, 2017.

[32] H. He, K. Yang, H. Liang, M. Menendez, and S. I. Guler, "Providing public transport priority in the perimeter of urban networks: A bimodal strategy," Transportation Research Part C: Emerging Technologies, vol. 107, pp. 171 - 192, 2019.

[33] G. Mariotte and L. Leclercq, "Flow exchanges in multi-reservoir systems with spillbacks," Transportation Research Part B: Methodological, vol. 122, pp. $327-349,2019$.

[34] W. Vickrey, "Congestion in midtown manhattan in relation to marginal cost pricing," Economics of Transportation, vol. 21, p. 100152, 2020.

[35] R. Arnott, "A bathtub model of downtown traffic congestion," Journal of Urban Economics, vol. 76, pp. 110-121, 2013.

[36] R. Lamotte and N. Geroliminis, "The morning commute in urban areas: Insights from theory and simulation," in Transportation Research Board 95th Annual Meeting., 2016.

[37] G. Mariotte, L. Leclercq, and J. A. Laval, "Macroscopic urban dynamics: Analytical and numerical comparisons of existing models," Transportation Research Part B, vol. 101, pp. 245-267, 2017.

[38] L. Leclercq, A. Sénécat, and G. Mariotte, "Dynamic macroscopic simulation of on-street parking search: A trip-based approach," Transportation Research Part B: Methodological, vol. 101, pp. 268-282, 2017.

[39] R. Quirynen, K. Berntorp, and S. Di Cairano, "Embedded optimization algorithms for steering in autonomous vehicles based on nonlinear model predictive control," in 2018 Annual American Control Conference (ACC). IEEE, 2018, pp. 3251-3256.

[40] H. Mittelmann. (2019) Decision tree for optimization software. [Online]. Available: http://plato.asu.edu/guide.html

[41] J. Nocedal and S. Wright, Numerical optimization. Springer Science \& Business Media, 2006.

[42] D. Simon, Optimal state estimation: Kalman, $H$ infinity, and nonlinear approaches. John Wiley \& Sons, 2006.

[43] J. Prakash, A. P. Deshpande, and S. C. Patwardhan, "State estimation and fault tolerant nonlinear predictive control of an autonomous hybrid system using unscented kalman filter," in Nonlinear model predictive control. Springer, 2009, pp. 285-293.

[44] T. Seo, A. M. Bayen, T. Kusakabe, and Y. Asakura, "Traffic state estimation on highway: A comprehensive survey," Annual reviews in control, vol. 43, pp. 128-151, 2017.

[45] A. Kouvelas, M. Saeedmanesh, and N. Geroliminis, "Linear-parametervarying model predictive control for multi-region traffic systems," in Transportation Research Board $98^{\text {th }}$ Annual Meeting., 2019.

[46] M. Saeedmanesh, A. Kouvelas, and N. Geroliminis, "A real-time state estimation approach for multi-region mfd traffic systems based on extended kalman filter," in Transportation Research Board $98^{\text {th }}$ Annual Meeting., 2019.

[47] K. György, A. Kelemen, and L. Dávid, "Unscented kalman filters and particle filter methods for nonlinear state estimation," Procedia Technology, vol. 12, pp. 65-74, 2014.

[48] S. Subramanian, S. Lucia, and S. Engell, "Economic multi-stage output feedback nmpc using the unscented kalman filter," IFAC-PapersOnLine, vol. 48 , no. 8 , pp. $38-43,2015$.

[49] L. Leclercq, "Hybrid approaches to the solutions of the "lighthillwhitham-richards" model," Transportation Research Part B: Methodological, vol. 41, pp. 701-709, 2007.

[50] J. M. Maciejowski, Predictive control: with constraints. Pearson education, 2002.

[51] S. F. A. Batista and L. Leclercq, "Regional dynamic traffic assignment framework for mfd multi-regions models," Transportation Science, vol. 53, pp. 1563-1590, 2019.

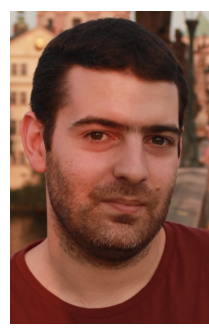

S. F. A. Batista graduated in Astrophysics and Mathematical Engineering from the Faculty of Sciences, University of Porto (Portugal). He has received a $\mathrm{Ph} . \mathrm{D}$. degree in Civil Engineering (focusing on transportation) from the University of Lyon (France). $\mathrm{He}$ is currently a Post-Doctoral Associate at the Division of Engineering of New York University Abu Dhabi (United Arab Emirates). His main research interests focus on traffic flow models, sustainable and smart cities, urban traffic control and demand management.

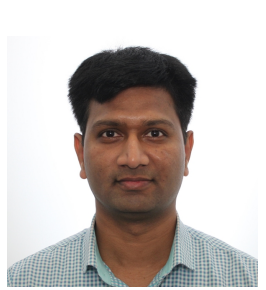

Deepak Ingole received an M.Tech degree in Instrumentation and Control Engineering from $\mathrm{COE}$ Pune, in 2012. In 2014, he was selected as an MSCA fellow under the TEMPO project at STU in Bratislava, Slovakia, where he received a Ph.D. degree in Process Control Engineering in 2017. During his Ph.D. he worked at the University of Oxford and Imperial College London as a visiting researcher. He served as a postdoc during 2017-2020 under the ERC project MAGnUM at the University of Gustave Eiffel. He is currently, a postdoc under the Moonshot-FLEX project at the KU Leuven, Belgium. His research interests include MPC, optimization, embedded systems, and the control of transportation, biomedical, and processing systems.

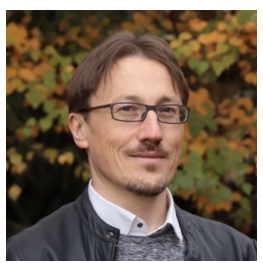

Ludovic Leclercq is a Professor at Universit Gustave Eiffel. He received his engineering and master degrees in Civil Engineering in 1998, his Ph.D. in 2002 and his habilitation thesis (HDR) in 2009. He is currently deputy director of the LICIT laboratory, a joint research unit from University Gustave Eiffel and ENTPE, and head of a research group about traffic modeling and analysis. His research interests correspond to multi-scale and multi-modal dynamic traffic modeling. Smart cities, mobility as a service, sustainable and reliable transportation systems are some of the applications his researches are targeting. He is a member of the multiple editorial boards of Transportation journals and also a member of the TRB committee Traffic Flow Theory and Characteristics and the international advisory committee of ISTTT. In 2015, he was awarded the most prestigious research grant in Europe, i.e., an ERC consolidator grant in Social Science and Humanities.

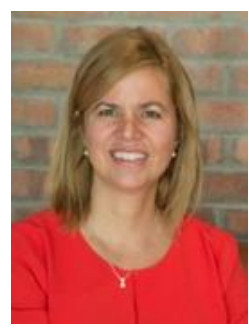

Mónica Menéndez received the dual B.S. degree (summa cum laude) in civil and architectural engineering from University of Miami, FL, USA, in 2002 and the M.S. and Ph.D. degrees (focusing on transportation) from University of California, Berkeley, CA, USA, in 2003 and 2006, respectively. Since January 2018, she is an Associate Professor of Civil and Urban Engineering at New York University in Abu Dhabi; and a Global Network Associate Professor of Civil and Urban Engineering at the Tandon School of Engineering in New York University. Between 2010 and 2017, she was the Director of the research group Traffic Engineering at ETH Zurich; and prior to that, a Management Consultant at Bain \& Company. She has authored or coauthored over 65 journal articles and over 160 conference contributions and technical reports in the area of transportation. Her research interests include multimodal transportation systems paying special attention to new technologies and information sources. Prof. Menendez is an active reviewer for over 20 journals, and a member of multiple editorial boards for top journals in Transportation, including IEEE Transactions or ITS. 